\title{
Long non-coding RNA HOTAIR overexpression improves premature ovarian failure by upregulating Notch-1 expression
}

\author{
WEI ZHAO and LIWEI DONG
}

Department of Reproductive Medicine, People's Hospital of Dezhou, Dezhou, Shandong 253014, P.R. China

Received February 2, 2018; Accepted May 17, 2018

DOI: $10.3892 /$ etm.2018.6750

\begin{abstract}
The aim of the present study was to investigate the role of long non-coding (lnc)RNA HOTAIR in premature ovarian failure. A total of 69 women with spontaneous premature ovarian failure and 48 healthy women were enrolled in the present study. Ovarian tissues and blood samples were harvested from each participant. The expression of HOTAIR in ovarian tissues and sera was detected using reverse transcription-quantitative polymerase chain reaction. A receiver operator characteristic curve analysis was performed to assess the diagnostic value of HOTAIR expression in ovarian tissues and sera for premature ovarian failure. Hamster ovary cell lines overexpressing HOTAIR were established and cell apoptosis were assessed using an MTT assay. The effect of HOTAIR upregulation on the expression of Notch-1 was measured using western blotting. The expression of HOTAIR in ovarian tissues and serum samples was significantly lower in patients with premature ovarian failure compared with healthy controls. These results suggest that ovarian and serum HOTAIR expression levels may be used to accurately predict the risk of premature ovarian failure. It was also demonstrated that HOTAIR overexpression upregulates Notch-1 protein expression in hamster ovary cells and reduced apoptosis, whereas the Notch inhibitor L685458 ameliorated these effects. In conclusion, the results of the present study suggest that LncRNA HOTAIR overexpression improves premature ovarian failure by upregulating the expression of Notch-1.
\end{abstract}

\section{Introduction}

Premature ovarian failure refers to the occurrence of hypergonadotropic hypogonadism, amenorrhea and infertility in females $<40$ years of age (1). It is thought that $1-3 \%$ of females will be affected by this disease in their lifetime (2). Ovarian

Correspondence to: Dr Liwei Dong, Department of Reproductive Medicine, People's Hospital of Dezhou, 1751 Xinhu Street, Dezhou, Shandong 253014, P.R. China

E-mail: fguu29i8g29@163.com

Key words: premature ovarian failure, long non-coding RNA HOTAIR, diagnosis, Notch-1, apoptosis transplantation between monozygotic twins is an effective treatment for premature ovarian failure; however, the clinical application of this technique is difficult due to the limited supply of ovarian tissues (3). One of the main challenges in treating premature ovarian failure is that its pathogenesis remains unclear (4). As such, focused research into the underlying mechanism responsible for the pathogenesis of premature ovarian failure is required to develop more effective treatments for this disease.

Long non-coding (lnc)RNAs are a group of non-coding RNAs $>200 \mathrm{nt}$ in length that serve important roles in the pathogenesis of various diseases (5). The functionality of lncRNA HOTAIR has been well studied in malignant tumor models (6), while the development and recovery of premature ovarian failure has been reported to be associated with certain lncRNAs (7). LncRNA HOTAIR is upregulated in ovarian cancer and serves a role in the regulation of tumor growth and metastasis $(8,9)$. However, the functionality of lncRNA HOTAIR in patients with premature ovarian failure has not yet been reported. The aim of the present study was to investigate the role of IncRNA HOTAIR in premature ovarian failure. The results revealed that IncRNA HOTAIR overexpression may improve premature ovarian failure by upregulating the expression of Notch-1. The present study provides a further insight into the involvement of HOTAIR in ovarian diseases and suggests a potential target for the treatment of premature ovarian failure.

\section{Materials and methods}

Patients. A total of 69 women with spontaneous premature ovarian failure were recruited at the Department of Reproductive Medicine, People's Hospital of Dezhou (Dezhou, China) between January 2015 and January 2017. Premature ovarian failure was defined as a follicle stimulating hormone result of $>30$ IU/1 when measured twice with an interval of 4 weeks (10). Patient age ranged from 19 to 40 years, with a mean age of $29.1 \pm 4.3$ years. Patients with other ovarian diseases or patients that had been treated in other hospitals prior to admission to the People's Hospital of Dezhou were excluded. A total of 48 healthy women (age range, 21-40 years; mean age, 29.8 \pm 5.1 years) were enrolled as a control group. The present study was approved by the Ethics Committee of the People's Hospital of Dezhou and all participants provided signed informed consent. 
Total RNA extraction. Ovarian tissues were harvested from each participant via a fine needle aspiration biopsy. Fasting blood samples were collected on the morning after admission. Blood samples were stored at room temperature for $2 \mathrm{~h}$, followed by centrifugation at $1,200 \mathrm{xg}$ for $20 \mathrm{~min}$ at room temperature to collect the serum. TRIzol (Invitrogen; Thermo Fisher Scientific, Inc., Waltham, MA, USA) was used to extract total RNA from serum samples and ovarian tissues. Ovarian tissues were ground in liquid nitrogen prior to the addition of TRIzol.

Reverse transcription-quantitative polymerase chain reaction (RT-qPCR). A NanoDrop ${ }^{\mathrm{TM}} 2000$ Spectrophotometer (Thermo Fisher Scientific, Inc.) was used to measure the quality of RNA samples. Samples with an A260/A280 ratio between 1.8 and 2.0 were subjected to RT for cDNA synthesis using an iScript ${ }^{\mathrm{TM}}$ cDNA Synthesis kit (Bio-Rad Laboratories, Inc., Hercules, CA, USA). The PCR reaction system was prepared using SYBR ${ }^{\circledR}$ Green Real-Time PCR Master mix and performed on an ABI 7500 System (both from Thermo Fisher Scientific, Inc.). Primers used were as follows: HOTAIR, forward 5'-GGT CCTGCTCCGCTTCGCAG-3' and reverse 5'-ACGCCCCTC CTTCCTCTCGC-3'; $\beta$-actin, forward 5'-GACCTCTATGCC AACACAGT-3' and reverse 5'-AGTACTTGCGCTCAGGAG GA-3'. Thermocycling conditions were as follows: $95^{\circ} \mathrm{C}$ for $55 \mathrm{sec}$ followed by 40 cycles of $95^{\circ} \mathrm{C}$ for $15 \mathrm{sec}$ and $60^{\circ} \mathrm{C}$ for $50 \mathrm{sec}$. Data were quantified using the $2^{-\Delta \Delta \mathrm{Cq}}$ method (11). The relative expression of HOTAIR was normalized to $\beta$-actin.

Cells and culture. The normal hamster ovarian cell lines Lec8 (cat. no. CRL-1737 ${ }^{\mathrm{TM}}$ ) and CHO (cat. no. CRL-9096 ${ }^{\mathrm{TM}}$ ) were obtained from the American Type Culture Collection (Manassas, VA, USA). Lec8 cells were cultured in $\alpha$ minimum essential medium (Gibco; Thermo Fisher Scientific, Inc.) supplemented with $10 \%$ fetal bovine serum (FBS, Sigma-Aldrich; Merck KGaA, Darmstadt, Germany). CHO cells were cultured with Iscove's modified Dulbecco's medium (Sigma-Aldrich; Merck KGaA) supplemented with 10\% FBS. All cells were cultured in an atmosphere containing $5 \% \mathrm{CO}_{2}$ at $37^{\circ} \mathrm{C}$. Cells in the logarithmic growth phase were harvested for subsequent experiments.

Construction of HOTAIR overexpression cell lines. HOTAIR cDNA was amplified from cDNA of ovarian tissues using primers (Shanghai GenePharma Co., Ltd., Shanghai, China) carrying EcoRI restriction enzyme sites at the flanking ends. The EcoRI-EcoRI fragment containing HOTAIR cDNA was inserted into GV299 lentiviral vectors (Shanghai GeneChem Co., Ltd.) to establish HOTAIR overexpression vector. Lec8 and $\mathrm{CHO}$ cells were cultured overnight until 80-90\% confluence was reached, following which they were transfected with vectors using Lipofectamine ${ }^{\circledR} 2000$ reagent (Invitrogen; Thermo Fisher Scientific, Inc.). Empty vectors without HOTAIR cDNA were used as negative controls. Cells were cultured in an atmosphere containing $5 \% \mathrm{CO}_{2}$ at $37^{\circ} \mathrm{C}$ for $24 \mathrm{~h}$ prior to subsequent experiments.

MTT assay. Lec8 and CHO cells were harvested and single cell suspensions were prepared using RPMI1640 (Gibco; Thermo Fisher Scientific, Inc.). Cell suspensions were diluted to give a final cell density of $5 \times 10^{4}$ cells $/ \mathrm{ml}$. Notch was inhibited by $1 \mu \mathrm{g} / \mathrm{ml}$ L685458 (Sigma-Aldrich; Merck KGaA). A total of $100 \mu \mathrm{l}$ cell suspension was seeded in each well of a 96-well plate. Cells were incubated at $37^{\circ} \mathrm{C}$ for $8 \mathrm{~h}$, followed by the addition of $10 \mu \mathrm{l}$ MTT (Sigma-Aldrich; Merck KGaA) in each well. Following incubation for $4 \mathrm{~h}$ at $37^{\circ} \mathrm{C}$, formazan was dissolved by adding dimethyl sulfoxide. Absorbance was measured at $570 \mathrm{~nm}$ using a microtiter plate reader. Values were normalized to control cells.

Western blotting. Total proteins were extracted from Lec8 and $\mathrm{CHO}$ cells using radioimmunoprecipitation assay buffer (Thermo Fisher Scientific, Inc.). Protein samples were quantified using the BCA method. A total of $30 \mu \mathrm{g}$ protein/lane was separated by $10 \%$ SDS-PAGE and transferred onto polyvinylidene fluoride membranes. Blocking was performed by incubating with $5 \%$ skimmed milk at room temperature for $1 \mathrm{~h}$. Membranes were washed in TBST (3\% Tween-20) and incubated with primary antibodies, including rabbit anti-Notch1 antibody (1:2,000; ab65297) and anti-GAPDH antibody (1:1,000; ab9485) (both from Abcam, Cambridge, $\mathrm{UK})$, overnight at $4^{\circ} \mathrm{C}$. Membranes were washed with TBST (3\% Tween-20) and further incubated with anti-rabbit horseradish peroxidase-conjugated immunoglobulin $\mathrm{g}$ secondary antibodies (1:1,000; MBS435036; MyBioSource, San Diego, CA, USA) at room temperature for $1 \mathrm{~h}$. Membranes were subsequently washed with TBST (3\% Tween-20) and signals were detected using enhanced chemiluminescence (Sigma-Aldrich; Merck KGaA). The relative expression of Notch-1 was normalized to endogenous control GAPDH using ImageJ (version 1.37; National Institutes of Health, Bethesda, MD, USA).

Statistical analysis. SPSS 19.0 (IBM Corp., Armonk, NY, USA) was used for all statistical analyses and data are expressed as the mean \pm standard deviation. Comparisons between two groups were made using t tests and multiple group comparisons were made using one-way analysis of variance and the least significant difference post hoc test. Receiver operator characteristic (ROC) analysis was used to evaluate the diagnostic value of HOTAIR expression for premature ovarian failure and data was reported as the mean percentage and 95\% confidence interval (CI). The cut-off value for HOTAIR in ovarian tissues was 0.1221 . The cut-off value for HOTAIR in serum was 0.1140 . $\mathrm{P}<0.05$ was considered to indicate a statistically significant differences.

\section{Results}

HOTAIR expression in ovarian tissues from patients with premature ovarian failure and healthy controls. HOTAIR expression in ovarian tissues from patients with premature ovarian failure and healthy controls was assessed using RT-qPCR. The expression of HOTAIR was significantly decreased in patients with premature ovarian failure compared with the control group, indicating that HOTAIR downregulation may serve a role in the pathogenesis of premature ovarian failure (Fig. 1; $\mathrm{P}<0.05$ ).

Serum HOTAIR expression in patients with premature ovarian failure and healthy controls. Serum HOTAIR expression was 
significantly lower in patients with premature ovarian failure compared with the control group (Fig. 2; $\mathrm{P}<0.05$ ).

Diagnostic value of HOTAIR expression in ovarian tissues and sera for premature ovarian failure. ROC analysis was performed to evaluate the diagnostic value of serum and ovarian tissue HOTAIR expression for premature ovarian failure. The area under the curve (AUC) for ovarian tissue HOTAIR expression in the diagnosis of premature ovarian failure was 0.9119, with a 95\% CI of 0.8620-0.9619 (Fig. 3A; $\mathrm{P}<0.0001)$. Furthermore, the AUC for serum HOTAIR in the diagnosis of premature ovarian failure was 0.8868 , with a 95\% CI of 0.8295-0.9441 ( $\mathrm{P}<0.0001$; Fig. 3B). These results suggest that HOTAIR expression in the ovarian tissues and serum can be used to accurately predict the risk of premature ovarian failure. Although the diagnostic accuracy of HOTAIR expression in ovarian tissues is higher than that of serum HOTAIR, measuring serum HOTAIR is less invasive.

HOTAIR overexpression promotes Notch-1 expression in two normal hamster ovarian cell lines. It has been reported that lncRNA HOTAIR is able to activate the Notch signaling pathway (12). In the present study, HOTAIR overexpression was induced in hamster ovarian cells and Notch-1 expression was assessed using western blotting. No significant differences were observed in the expression of Notch-1 protein between control (untransfected) cells and negative control (transfected with empty vectors) cells (Fig. 4). However, the expression of Notch-1 protein was significantly increased in HOTAIR-overexpressing Lec8 and CHO cells compared with the control and negative control groups ( $\mathrm{P}<0.05$; Fig. 4). These data suggest that HOTAIR overexpression is able to upregulate the expression of Notch-1.

HOTAIR overexpression inhibits the apoptosis of hamster ovarian cells. Notch-1 overexpression has been reported to inhibit the apoptosis of various cancers, while activation of Notch-1 signaling has been demonstrated to be an effective treatment for premature ovarian failure (13). In the present study, HOTAIR overexpression significantly inhibited the apoptosis of hamster ovarian cells compared with control cells ( $\mathrm{P}<0.05$; Fig. 5). However, treatment with Notch inhibitor L685458 (a Notch inhibitor) significantly inhibited Notch-1 expression and increased cell apoptosis $(\mathrm{P}<0.05$; Fig. 5). These results suggest that Notch-1 overexpression is able to inhibit the apoptosis of ovarian cells, possibly by activating the Notch signaling pathway.

\section{Discussion}

Abnormal lncRNA HOTAIR expression has been reported in the development of various diseases $(8,9)$. In a gastric cancer study, the expression of HOTAIR was demonstrated to be upregulated in tumor tissues compared with adjacent healthy tissues and it was revealed that HOTAIR promoted cancer development and increased chemoresistance cisplatin (14). LncRNA HOTAIR overexpression has also been observed in liver cancer and was demonstrated to be responsible for the increased growth rate of tumor cells (15). Furthermore,

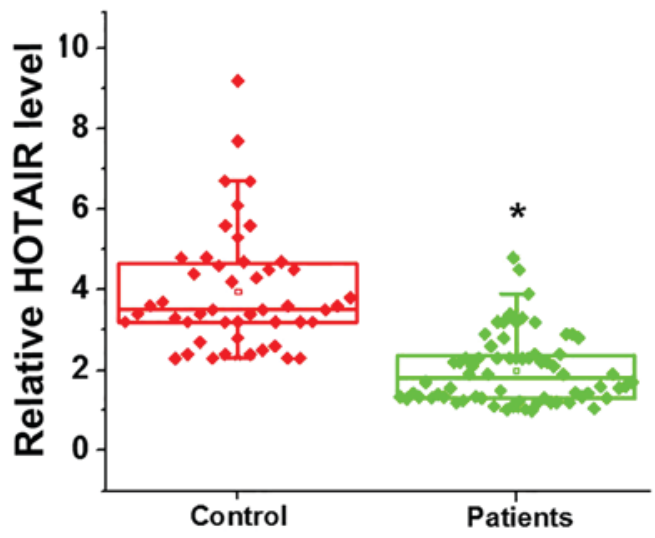

Figure 1. HOTAIR expression in ovarian tissues from patients with premature ovarian failure and healthy controls. ${ }^{*} \mathrm{P}<0.05$ vs. control.

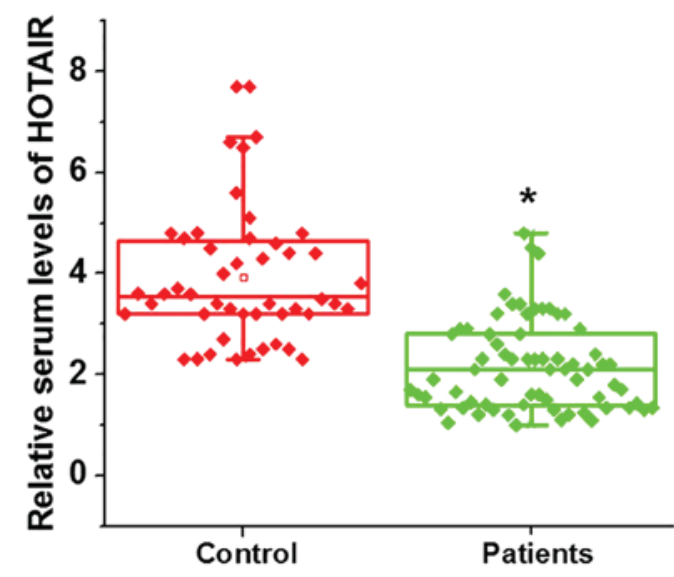

Figure 2. Serum HOTAIR expression in patients with premature ovarian failure and healthy controls. ${ }^{*} \mathrm{P}<0.05$ vs. control.

HOTAIR overexpression is associated with the development of ovarian cancer (16). In the present study, HOTAIR expression was demonstrated to be significantly reduced in ovarian tissues and serum samples from patients with premature ovarian failure compared with healthy controls. These results indicate that IncRNA HOTAIR downregulation may serve a role in the pathogenesis of premature ovarian failure.

The occurrence and development of disease is typically accompanied by changes in the blood, and so blood testing is performed to aid diagnosis (17). A previous study reported that serum levels of anti-Müllerian hormone are correlated with early ovarian aging in young and infertile women; these levels can be used to predict accelerated oocyte loss, but not unfavorable pregnancy outcomes (18). To the best of our knowledge, no accurate and effective biomarker for premature ovarian failure has previously been reported. In the present study, ROC curve analysis revealed that HOTAIR expression in ovarian tissues and sera could accurately predict the risk of premature ovarian failure. Although the diagnostic accuracy of HOTAIR expression in ovarian tissues was higher compared with serum HOTAIR, serum HOTAIR is easier to measure using less invasive techniques. HOTAIR expression is generally upregulated in ovarian diseases, including cancer (16), and so HOTAIR downregulation may be used 

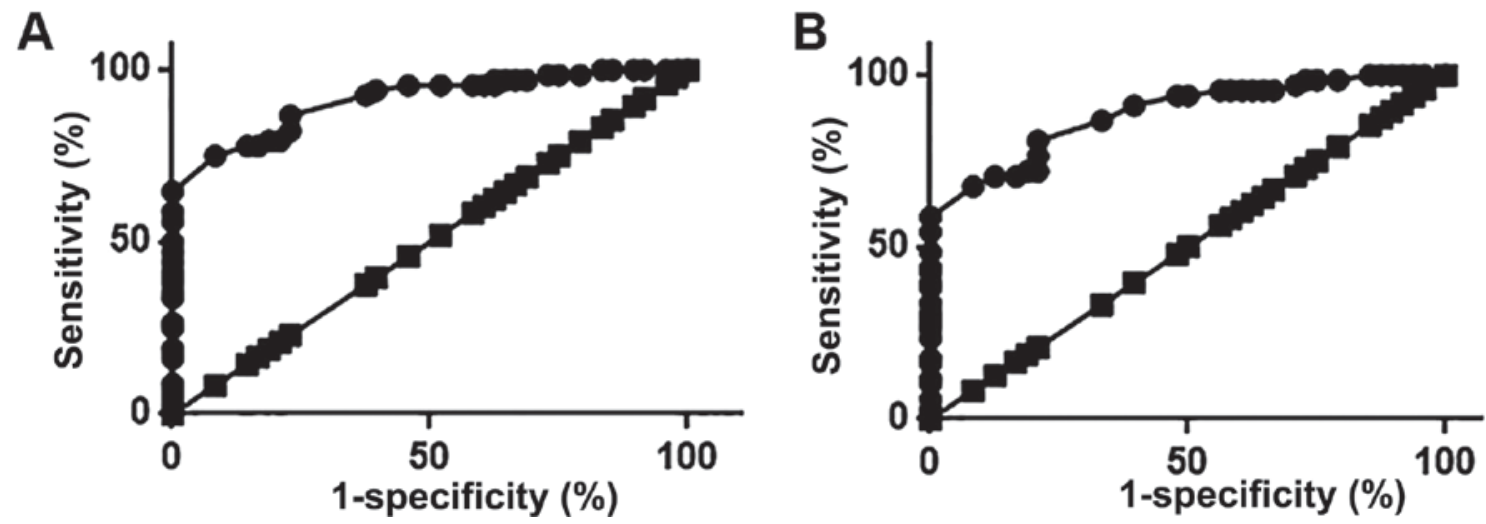

Figure 3. Diagnostic value of HOTAIR expression. Diagnostic value of HOTAIR expression in (A) ovarian tissues and (B) serum for premature ovarian failure. HOTAIR expression data were obtained through reverse transcription-quantitative polymerase chain reaction. The true positive rate (sensitivity) was plotted against the false positive rate (1-specificity). The cut-off value for HOTAIR in ovarian tissues was 0.1221 . The cut-off value for HOTAIR in serum was 0.1140 . Solid circles represent diagnostic curve. Squares represent the line of identity.

to distinguish premature ovarian failure from other ovarian diseases.

The results of a previous study revealed that activation of the Notch-1 signaling pathway is important for the recovery of premature ovarian failure in mice (13). It is also well known that HOTAIR interacts with the Notch pathway to exert its biological functions (19). In the present study, Notch-1 was significantly increased in cells overexpressing HOTAIR, suggesting that HOTAIR may also serve a role in premature ovarian failure by interacting with the Notch pathway. Notch-1 overexpression has previously been demonstrated to inhibit apoptosis in certain cells (20), while Notch-1 downregulation inhibits cell growth and promotes apoptosis (21). In the present study, HOTAIR overexpression significantly inhibited the apoptosis of hamster ovarian cells, while treatment with Notch inhibitor significantly increased cell apoptosis. These results suggest that Notch-1 overexpression may ameliorate premature ovarian failure by inhibiting the apoptosis of ovarian cells, at least in part via activation of the Notch signaling pathway.

In conclusion, HOTAIR expression is downregulated in ovarian tissues and serum samples from patients with premature ovarian failure compared with healthy controls. Both ovarian tissues and sera may be used to accurately predict the risk of premature ovarian failure. HOTAIR overexpression promotes Notch-1 protein expression and inhibits apoptosis in hamster ovarian cells, effects that are reversed by treatment with Notch inhibitor L685458. In conclusion, the results of the present study suggest that lncRNA HOTAIR overexpression is able to improve premature ovarian failure by upregulating the expression of Notch-1. The present study is limited due to the small sample size and further research is required to confirm these results.

\section{Acknowledgements}

Not applicable.

\section{Funding}

No funding was received.
A

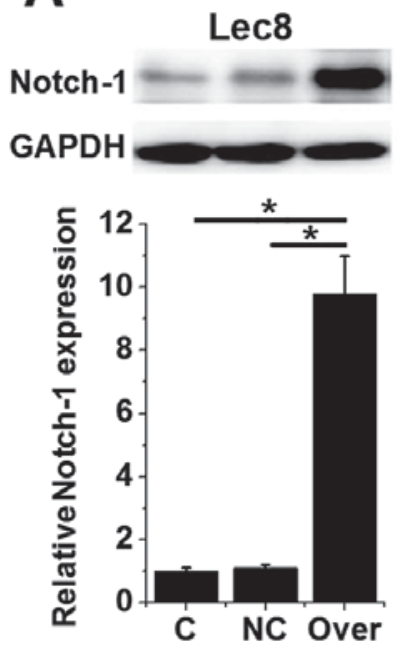

B

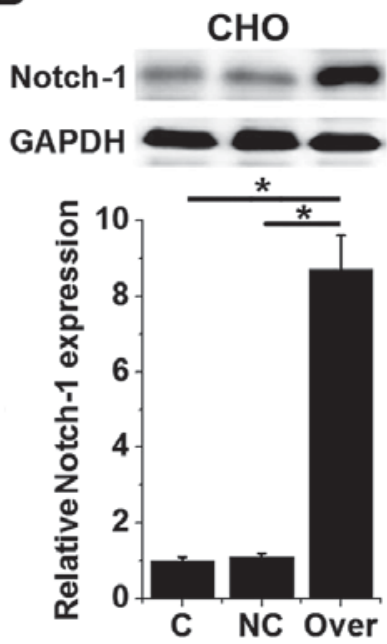

Figure 4. HOTAIR overexpression promoted Notch-1 protein expression in (A) Lec8 and (B) CHO normal hamster ovary cell lines. ${ }^{*} \mathrm{P}<0.05$. $\mathrm{C}$, non-transfected cells; NC, negative control (cells transfected with empty vectors); Over, overexpression.

\section{Availability of data and materials}

The datasets used and/or analyzed during the present study are available from the corresponding author upon reasonable request.

\section{Authors' contributions}

WZ and LD conceived and designed the study. WZ performed experiments and analyzed data. LD interpreted the data and drafted the manuscript. Both authors read and approved the final manuscript.

\section{Ethics approval and consent to participate}

The present study was approved by the Ethics Committee of People's Hospital of Dezhou and all participants provided signed informed consent. 

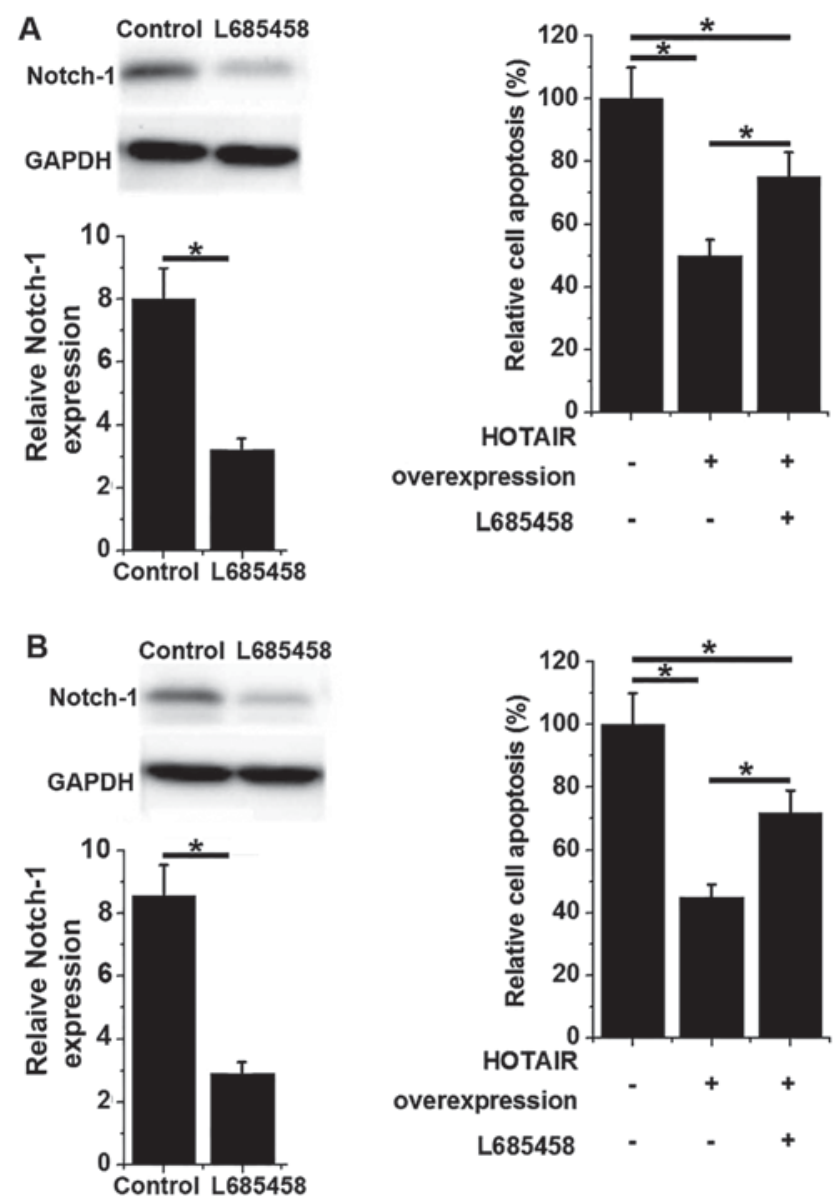

Figure 5. Apoptosis of hamster ovary cells under different conditions. Notch-1 expression in (A) Lec8 and (B) CHO cells overexpressing HOTAIR and treated with or without Notch inhibitor L685458. ${ }^{*} \mathrm{P}<0.05$.

\section{Patient consent for publication}

Signed informed consent was obtained from all participants and/or guardians.

\section{Competing interests}

The authors declare that they have no competing interests.

\section{References}

1. Jankowska K: Premature ovarian failure. Prz Menopauzalny 16: 51-56, 2017.

2. Kovanci E and Schutt AK: Premature ovarian failure: Clinical presentation and treatment. Obstet Gynecol Clin North Am 42: 153-161, 2015.

3. Silber SJ, Lenahan KM, Levine DJ, Pineda JA, Gorman KS, Friez MJ, Crawford EC and Gosden RG: Ovarian transplantation between monozygotic twins discordant for premature ovarian failure. N Engl J Med 353: 58-63, 2005.

4. Luisi S, Orlandini C, Regini C, Pizzo A, Vellucci F and Petraglia F: Premature ovarian insufficiency: From pathogenesis to clinical management. J Endocrinol Invest 38: 597-603, 2015.

5. Yang X, Gao L, Guo X, Shi X, Wu H, Song F and Wang B: A network based method for analysis of lncRNA-disease associations and prediction of IncRNAs implicated in diseases. PLoS One 9: e87797, 2014.

6. Cai B, Song XQ, Cai JP and Zhang S: HOTAIR: A cancer-related long non-coding RNA. Neoplasma 61: 379-391, 2014.
7. Xiong Y, Liu T, Wang S, Chi $\mathrm{H}$, Chen $\mathrm{C}$ and Zheng J: Cyclophosphamide promotes the proliferation inhibition of mouse ovarian granulosa cells and premature ovarian failure by activating the lncRNA-Meg3-p53-p66Shc pathway. Gene 596: $1-8,2017$

8. Qiu JJ, Lin YY, Ye LC, Ding JX, Feng WW, Jin HY, Zhang Y, Li Q and Hua KQ: Overexpression of long non-coding RNA HOTAIR predicts poor patient prognosis and promotes tumor metastasis in epithelial ovarian cancer. Gynecol Oncol 134: 121-128, 2014.

9. Qiu JJ, Wang Y, Ding JX, Jin HY, Yang G and Hua KQ: The long non-coding RNA HOTAIR promotes the proliferation of serous ovarian cancer cells through the regulation of cell cycle arrest and apoptosis. Exp Cell Res 333: 238-248, 2015.

10. Cartwright B, Robinson J, Seed PT, Fogelman I and Rymer J: Hormone replacement therapy versus the combined oral contraceptive pill in premature ovarian failure: A randomized controlled trial of the effects on bone mineral density. J Clin Endocrinol Metab 101: 3497-3505, 2016.

11. Livak KJ and Schmittgen TD: Analysis of relative gene expression data using real-time quantitative PCR and the 2(-Delta Delta C(T)) method. Methods 25: 402-408, 2001.

12. Dong C, Liu S, Lv Y, Zhang C, Gao H, Tan L and Wang H: Long non-coding RNA HOTAIR regulates proliferation and invasion via activating Notch signalling pathway in retinoblastoma. J Biosci 41: 677-687, 2016.

13. Liu TE, Wang S, Zhang L, Guo L, Yu Z, Chen C and Zheng J: Growth hormone treatment of premature ovarian failure in a mouse model via stimulation of the Notch-1 signaling pathway. Exp Ther Med 12: 215-221, 2016.

14. Yan J, Dang Y, Liu S, Zhang Y and Zhang G: LncRNA HOTAIR promotes cisplatin resistance in gastric cancer by targeting miR-126 to activate the PI3K/AKT/MRP1 genes. Tumor Biol 37: 16345-16355, 2016.

15. Li H, An J, Wu M, Zheng Q, Gui X, Li T, Pu H and Lu D: LncRNA HOTAIR promotes human liver cancer stem cell malignant growth through downregulation of SETD2. Oncotarget 6: 27847-27864, 2015.

16. Teschendorff AE, Lee SH, Jones A, Fiegl H, Kalwa M, Wagner W, Chindera K, Evans I, Dubeau L, Orjalo A, et al: HOTAIR and its surrogate DNA methylation signature indicate carboplatin resistance in ovarian cancer. Genome Med 7: 108, 2015.

17. Pankla R, Buddhisa S, Berry M, Blankenship DM, Bancroft GJ, Banchereau J, Lertmemongkolchai $G$ and Chaussabel D: Genomic transcriptional profiling identifies a candidate blood biomarker signature for the diagnosis of septicemic melioidosis. Genome Biol 10: R127, 2009.

18. Lin PY, Huang FJ, Kung FT, Chiang HJ, Lin YJ, Lin YC and Lan KC: Evaluation of serum anti-mullerian hormone as a biomarker of early ovarian aging in young women undergoing IVF/ICSI cycle. Int J Clin Exp Pathol 7: 6245-6253, 2014.

19. Lee M, Kim HJ, Kim SW, Park SA, Chun KH, Cho NH, Song YS and Kim YT: The long non-coding RNA HOTAIR increases tumour growth and invasion in cervical cancer by targeting the Notch pathway. Oncotarget 7: 44558-44571, 2016.

20. Shelly LL, Fuchs C and Miele L: Notch-1 inhibits apoptosis in murine erythroleukemia cells and is necessary for differentiation induced by hybrid polar compounds. J Cell Biochem 73: 164-175, 1999.

21. Wang Z, Zhang Y, Li Y, Banerjee S, Liao J and Sarkar FH: Down-regulation of Notch-1 contributes to cell growth inhibition and apoptosis in pancreatic cancer cells. Mol Cancer Ther 5: 483-493, 2006. 